\title{
Structured Electron Beam Illumination: A New Control Over the Electron Probe Weird Probes and New Experiments
}

Vincenzo Grillo, ${ }^{1,2,3}$ Jordan S Pierce $^{3}$, Ebrahim Karimi, ${ }^{4}$, Tayler R Harvey ${ }^{3}$, Roberto Balboni ${ }^{5}$, Gian Carlo Gazzadi $^{1}$, Erfan Mafakheri ${ }^{6}$, Federico Venturi ${ }^{6}$, Benjamin J McMorran ${ }^{3}$, Stefano Frabboni, ${ }^{1,6}$ and Robert W Boyd ${ }^{4,6}$

1. CNR-Istituto Nanoscienze, Centro S3, Via G. Campi 213/a, I-41125 Modena, Italy

2. CNR-IMEM Parco Area delle Scienze 37/A, I-43124 Parma, Italy

3. Department of Physics, University of Oregon, Eugene, 97403-1274 Oregon, USA

4. Department of Physics, University of Ottawa, 25 Templeton, Ottawa, Ontario, K1N 6N5 Canada

5. CNR-IMM Bologna, Via P. Gobetti 101, 40129 Bologna, Italy

6. Dipartimento FIM, Universitá di Modena e Reggio Emilia, Via G. Campi 213/a, I-41125 Modena, Italy

${ }^{7}$ Institute of Optics, University of Rochester, Rochester, New York 14627, USA

Since the introduction of electron vortex beams it has become increasingly clear the possibility to have an unprecedented control over the electron beams and to produce arbitrary electron beams [1][2][3]. Electron microscopy is basically rediscovering most of the progresses of optics.

In this field, researchers have been using holograms based on liquid crystal technology to produce any kind of light beam shape. These beams are, for example, at the basis of some of the most interesting fundamental experiments of quantum mechanics [4].

It is well known that the equations for electron and photons, in paraxial approximation, are formally the same; however, what really paved the road to holographic electron optics has been the possibility to nanofabricate patterns on SiN membranes to induce a controlled phase plate [5][6].

For the sake of example we reported in fig 1 some noticeable electron beam shapes like a) Ince-Gauss and b) Helix Ince Gauss beams. c) A beam obtained by the combination of two Laguerre Gaussian beams with opposite topological charge $+/-3$. All these beams are interesting because shape invariant upon propagation. Finally as an extreme example we demonstrate the possibility to shape the beam as the CNR institute logo.

By the use of different phase-retrieval methods or measuring the hologram characteristics we were able to characterize the generated wavefunction and to evaluate the quality of the generated electron beam. We verified that our results are comparable in terms of quality with optical holograms.

Beyond this basic analogy there are important added values in electron beams since 1) e-beams can reach atomic scale 2) electron possess charge.

This motivate the use of complex beams and illumination structuring as new tools for material investigation. As a first example we will show how the new electron optical elements can be used to increase in different ways the STEM resolution. Fig 2a, for example, shows an holographic spherical aberration corrector. Fig 2b is the experimental intensity and the calculated intensity and phase ( as hue color) of a Bessel beam (in this case $\mathrm{L}=2$ ) that can be also used for tomography and superresolution [3]. Fig 2c is the intensity and vortex phase of a beam with $L=200 \hbar$ that can be used for its very large magnetic moment to couple with material magnetism. Therefore in this talk the state of the art of beam structuring and a series of present and future applications will be shown. 


\section{References:}

[1] J. Verbeeck, H. Tian, and P. Schattschneider, Nature 467, (2010) p 301.

[2] B. J. McMorran, A. Agrawal, I. M. Anderson, A. A. Herzing, H. J. Lezec, J. J. McClelland, and J. Unguris, Science 331, 192 (2011).

[3] V. Grillo, E. Karimi, G C Gazzadi, S Frabboni,M R. Dennis, and R W. Boyd Phys. Rev. X 4, 011013 (2014)

[4] Alois Mair, Alipasha Vaziri, Gregor Weihs \& Anton Zeilinger Nature 412 (2001) 313

[5] V. Grillo, G C Gazzadi, E. Karimi, E Mafakheri,R W. Boyd, and S Frabboni Applied Physics Letters 104, 043109 (2014)

[6] T.R Harvey, J S Pierce1, A K Agrawal, P Ercius, M Linck and B J McMorran New Journal of Physics 16 (2014) 093039

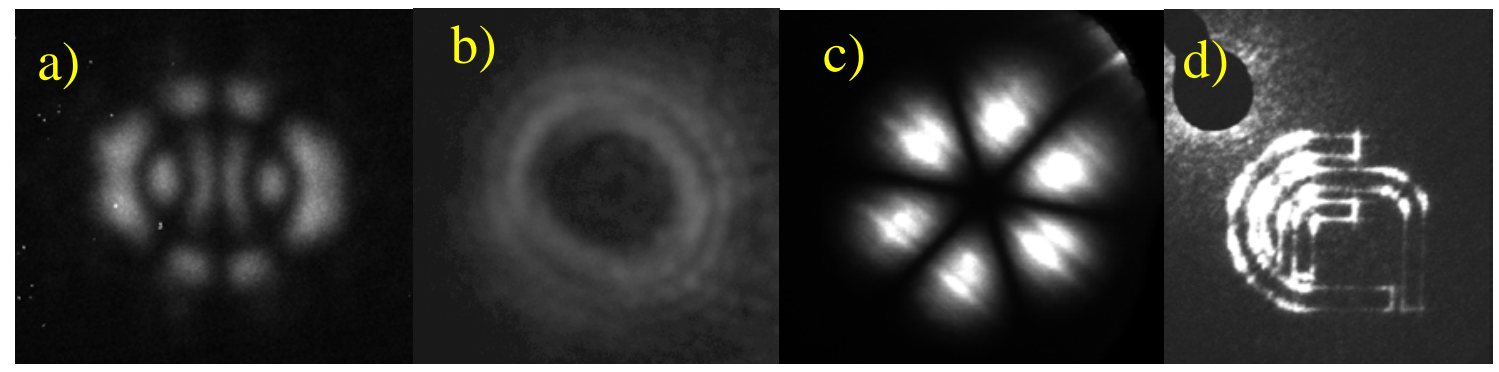

Figure 1. a,b,c) Intensity of different shape invariant beams a) Ince-Gauss b) Helix Ince Gauss c) Laguerre Gauss ( a combination of states with orbital number $= \pm 3$ ) . Additionally we show the ability on holographic shaping by producing a beam in the shape of the Logo of CNR.

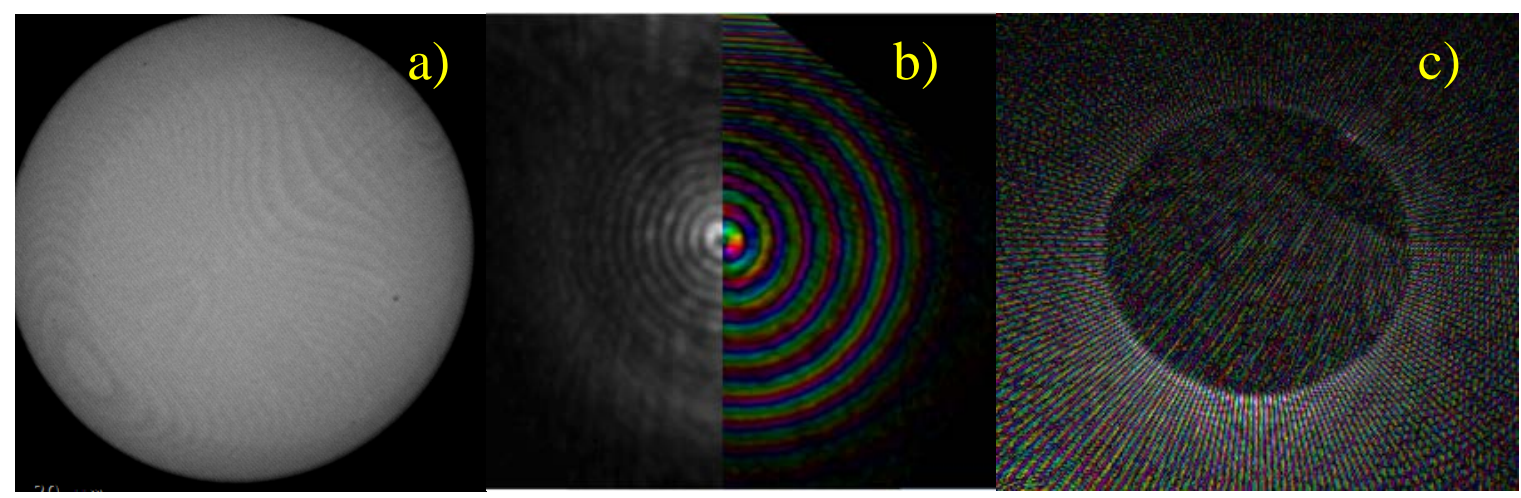

Figure 2. a) Hologram of a Spherical aberration corrector b) Tiled image of the experimental intensity (left) and calculated intensity/phase (right) for a Bessel beam L=2 c) Experimental image and calculated phase (after removing tilt effects) for a beam with Orbital angular momentum $\mathrm{L}=200$. The color-scales in fig b,c are such that brightness is related to wavefunction intensity and hue indicates the phase. 